ISSN 0103-9954

\title{
ABORDAGEM SISTÊMICA E APLICAÇÃO DE RUGOSIDADES PARA DESENCADEAR PROPRIEDADES EMERGENTES EM RESTAURAÇÃO DE SOLOS DEGRADADOS
}

\author{
SYSTEMIC APPROACH AND ROUGHNESS APPLICATION TO CAUSE EMERGING PROPERTIES \\ IN THE RESTORATION OF DEGRADED SOILS
}

\author{
Juarês José Aumond ${ }^{1}$ João Paulo de Maçaneiro²
}

\begin{abstract}
RESUMO
Baseado na Teoria Geral dos Sistemas desenvolveu-se um modelo ecológico para a restauração dos ecossistemas, cujos solos estejam muito degradados, tratando o ecossistema como um sistema dinâmico complexo, hipersensível às condições iniciais de preparação do solo. Partindo da hipótese de que os ecossistemas degradados são sensíveis às condições iniciais de preparação do solo, avaliou-se a técnica das rugosidades (variações do relevo, alternando superfícies côncavas e convexas) para desencadear, ao longo do tempo, propriedades emergentes que aceleram o processo de restauração ecológica. Os ecossistemas degradados podem ser entendidos como sistemas organizacionalmente abertos, como uma estrutura dissipativa, em que fluem matéria e energia, irreversivelmente. A principal tarefa, em restauração ecológica, em áreas que tiveram o solo degradado consiste em obter a internalização da matéria e da energia para induzir o sistema ao fechamento organizacional. As rugosidades, representadas pelas microtopografias do solo, constituem uma técnica eficiente na internalização de matéria, retendo água, sedimentos, matéria orgânica, nutrientes e sementes. As variações do relevo desencadeiam, ao longo do tempo, mudanças ambientais de uma forma dinâmica e heterogênea que influenciam as interações entre a radiação solar, a umidade e os nutrientes, criando oportunidades diferenciadas para as espécies vegetais e animais. Deve haver uma concentração orientada para as estruturas de fluxos e de processos existentes entre o ecossistema degradado (sistema) e o ambiente (vizinhança). Nessa abordagem dedica-se uma concentração especial nas inter-relações entre o sistema degradado e o ambiente. Para restauração ecológica, cuja área esteja com solo degradado, como a mineração e a pecuária, propõe-se uma nova abordagem sistêmica integradora na qual as rugosidades do solo possam desencadear espacial e temporalmente, padrões e propriedades ambientais emergentes devido à hipersensibilidade às condições iniciais de preparação do terreno.
\end{abstract}

Palavras-chave: restauração ecológica; pensamento sistêmico; internalização; microtopografias.

\begin{abstract}
Based on the general systems theory, an ecological model for the restoration of ecosystems has been developed, which soils are highly degraded, and treating the ecosystem as a complex dynamic system, hyper-sensitive to initial conditions of soil preparation. Assuming that degraded ecosystems are sensitive to initial conditions of soil preparation, the technique of roughness was evaluated (relief variations alternating between concave and convex surfaces) to trigger over time emergent properties that accelerate the process of ecological restoration. The degraded ecosystems can be understood as organizationally open systems, as a dissipative structure, in which irreversibly matter and energy flow. The main task in ecological restoration in areas that had the soil degraded is to achieve the internalization of matter and energy to induce the system to organizational closure. The roughness, represented by soil micro-topography is an effective technique in the internalization of matter, retaining water, sediment, organic matter, nutrients and seeds. Variations of relief trigger environmental changes over time in a dynamic and heterogeneous way, which influence

1 Geólogo, Dr., Professor do Departamento de Ciências Naturais, Universidade Regional de Blumenau, Rua Antônio da Veiga, 140, CEP 89012-900, Blumenau (SC), Brasil. aumond@furb.br

2 Engenheiro Florestal, Mestrando no Programa de Pós-Graduação em Engenharia Florestal, Departamento de Engenharia Florestal, Universidade Regional de Blumenau, Rua São Paulo, 3250, CEP 89012-900, Blumenau (SC), Brasil.jpmacaneiro@gmail.com
\end{abstract}

Recebido para publicação em 17/08/2011 e aceito em 25/03/2013

Ci. Fl., v. 24, n. 3, jul.-set., 2014 
the interactions between solar radiation, moisture and nutrients, creating different opportunities for plants and animal species. There must be an oriented concentration to flow structures and processes between the degraded ecosystem (system) and the environment (neighborhood). In this approach, a particular concentration on the interrelationships between the system and the environment is dedicated. For ecological restoration, whose area is with degraded soil, such as mining and ranching, a new integrative degraded systemic approach is proposed, in which the roughness of the soil might trigger spatial and temporal patterns and emergent environmental properties due to the hyper-sensitivity to initial conditions of the land preparation.

Keywords: ecological restoration; systems thinking; internalization; micro-topography.

\section{INTRODUÇÃO}

Nos últimos anos, a maior parte dos projetos de restauração ecológica foram executados por meio de técnicas reducionistas que comprometeram o processo de restauração, principalmente aqueles específicos que concentraram suas técnicas apenas no plantio de mudas (AUMOND, 2007; BRANCALION et al., 2010).

Aronson et al. (2011) identificam duas perspectivas diferentes entre ecólogos que trabalham em restauração de florestas tropicais no Brasil. Uma que defende instrumentos legais que definem um número mínimo de espécies nativas e uma proporção exata de grupos funcionais em projetos de restauração. Outro grupo defende que não há um know-how suficiente para estabelecer normas padronizadas e metodologias que possam justificar sua aplicação generalizada em projetos de restauração.

Uma análise mais detalhada da estratégia de uso da maior diversidade vegetal versus a estratégia de priorização e valorização das características funcionais das espécies vegetais dominantes na restauração dos ecossistemas e na produção primária, tratado por Naeem e Li (1997) e Grime (1997), respectivamente, evidenciam a complexidade do fenômeno da produção primária da biomassa na restauração dos ecossistemas naturais.

Grime (1997) defende as características funcionais das espécies vegetais dominantes como o principal e decisivo fator na recuperação e produção primária dos ecossistemas. No entanto, Naeem e Li (1997) defendem a diversidade vegetal como fator preponderante na produção de biomassa e recuperação dos ecossistemas. Ambos os enfoques diagnosticam aspectos distintos da dinâmica sucessional e da produtividade primária na restauração dos ecossistemas degradados (AUMOND, 2007).
A restauração ecológica deve restabelecer a estabilidade, estrutura (integridade biológica), função (saúde) e sustentabilidade dos ecossistemas naturais em longo prazo (SER, 2004; ENGEL e PARROTTA, 2008). O que deve ser priorizado num processo de restauração ecológica é o restabelecimento da estrutura e das funções do ecossistema original, sem focar demasiadamente na composição de espécies (MORAES et al., 2010). De acordo com Odum (1988), a estabilidade de um ecossistema relaciona-se mais intimamente com a diversidade funcional do que com a estrutural da biomassa existente.

Atualmente, não há estudos comprovando que a alta diversidade de espécies no plantio é suficiente para garantir, em todas as circunstâncias, o sucesso da restauração, ou seja, a estabilidade do ecossistema restaurado (DURIGAN et al., 2010). O fracasso e a frustração desses projetos de recuperação estão relacionados a uma visão antropocêntrica e utilitarista que remonta à primeira fase de manejo para a produção de florestas econômicas (AUMOND, 2003).

Estudos realizados por Anand e Desrochers (2004), utilizando modelos e conceitos de sistemas complexos para avaliar o processo de restauração ecológica, sugerem a validação da teoria do caos (Sistemas Dinâmicos Complexos) para compreender e quantificar os processos de restauração. Conceitos sobre atratores e padrões de comportamento das comunidades ao invés de focalizar medidas de espécies individuais e a análise dos componentes principais, embora seja uma técnica linear, podem ajudar a detectar trajetórias não lineares da restauração ecológica. Segundo experiências desses autores, duas fases da restauração foram detectadas numa área degradada, com base na teoria do caos. Uma fase linear inicial com a evolução da comunidade e consequente redução da área desmatada e uma segunda fase, 
na qual a restauração evolui em resposta a um atrator estranho na qual o seu estado final é difícil de se prever, mas que representa as circunstâncias necessárias para que o sistema ecológico siga sua complexa trajetória natural.

Os avanços conseguidos isoladamente em projetos de restauração ainda carecem de uma concepção multidisciplinar e de uma metodologia integradora. A nova estratégia deve basear-se no estado da inter-relação e interdependência essencial de todos os componentes e fenômenos físicos, químicos e biológicos que interagem no ecossistema degradado. Em sistemas muito degradados (i.e. áreas mineradas e utilizadas pela pecuária), recomendase a necessidade da internalização dos fatores ecológicos no sistema com auxílio das rugosidades (microtopografias) que funcionam como atratores gravitacionais (AUMOND, 2003; AUMOND, 2007; REGENSBURGER et al., 2008; AUMOND, 2009; AUMOND et al., 2012).

O que se propõe é que a restauração ecológica necessita de uma abordagem sistêmica, multidisciplinar e interdisciplinar, auxiliada por um modelo ecológico que envolva o máximo possível dos componentes abióticos e bióticos do ecossistema degradado.

\section{Embasamento teórico para a restauração ecológica através da internalização de matéria e energia}

Quanto mais estudamos um ecossistema degradado, mais somos levados a perceber que ele não pode ser entendido isoladamente. É preciso ter uma visão holística de todas as interações decorrentes do ecossistema degradado com seus componentes e do ambiente. Somente é possível concluir um processo de restauração ecológica quando ocorrer $\mathrm{o}$ fechamento organizacional de todos os componentes principais do sistema degradado (AUMOND, 2009).

Para entender as interações entre o ecossistema degradado e seus componentes é recomendável a utilização do pensamento sistêmico como ferramenta facilitadora na interpretação dos processos ecológicos (AUMOND, 2007; GRIFFITH, 2009).

O pensamento sistêmico trabalha com a mudança das partes para o todo. Um ecossistema degradado é uma totalidade integrada cujas propriedades não podem ser reduzidas às de partes menores. Suas propriedades essenciais, ou "sistêmicas", são propriedades do todo, que nenhuma das partes isoladamente possui. Elas surgem das relações de organização das partes que compõem o sistema degradado, isto é, de uma configuração de relações ordenadas que é característica do ecossistema degradado (CAPRA, 1996; GRIFFITH, 2005; AUMOND, 2009; GRIFFITH, 2009; SCHLINDWEIN, 2009). Algumas destas propriedades são destruídas e deixam de exercer sua função quando ocorre um distúrbio ou degradação no ecossistema.

Como todos os sistemas naturais, um ecossistema degradado pode ser visto, exatamente, como uma estrutura dissipativa, onde flui matéria e energia irreversivelmente (BERTALANFFY, 1975; PRIGOGINE e STENGER, 1984; PRIGOGINE e GLANSDORFF, 1997). Todos os ecossistemas naturais são abertos do ponto de vista do fluxo de matéria e energia e por isso é relevante, no conceito de ecossistema funcional, reconhecer que existe um ambiente de entrada e um ambiente de saída para manter os processos vitais em equilíbrio dinâmico (BERTALANFFY, 1975; ODUM, 1988).

Na concepção de Maciel (1974), um sistema (todo) é constituído de elementos (partes) e neles estão embutidos dois atributos: o conjunto das relações que ligam entre si os elementos e o conjunto de atividades desses elementos. Um sistema é um processo operacional global e implica sempre e simultaneamente a existência de três conjuntos: o conjunto de elementos, o conjunto de relações desses elementos e o conjunto de atividades efetivas ou potenciais. Para que haja um sistema suas partes devem estar interconectadas funcionando como um todo. $\mathrm{O}$ arranjo das partes do sistema é crucial e caso se elimine ou se extraia uma parte, então ele não funcionará como o sistema original. O comportamento do sistema depende de toda a estrutura e a mudança na estrutura implica na mudança do sistema (O’CONNOR, 1997).

Aumond (2007) entende floresta como um supersistema dissipativo, estruturalmente aberto ao fluxo de matéria e energia, porém, fechado organizacionalmente. Todos os seus componentes, plantas, animais, solo e sua macro, meso e microfauna, formam uma rede complexa de interações, caracterizando um conjunto de relações entre processos de produção que se mantêm e produz seus componentes dentro da cadeia alimentar, mantendo o conjunto afastado do estado de equilíbrio.

Segundo Hobbs e Harris (2001), um 
sistema natural não é fechado e estático, mas aberto e dinâmico. Nesta perspectiva, os ecossistemas naturais estão sujeitos a distúrbios, que podem ser entendidos como eventos naturais discretos (ENGEL e PARROTTA, 2008).

Souza e Buckeridge (2004) defendem que os sistemas biológicos não são apenas estruturalmente, mas também funcionalmente complexos. Para esses autores, sistemas complexos são aqueles compostos de muitos elementos e/ou subsistemas diferentes, interagindo espacial e temporalmente de forma não linear, gerando padrões emergentes. A complexidade dos sistemas pode estar relacionada com o grau de conectância, formando uma intrincada rede de relações. Os sistemas naturais são sistemas complexos e fica evidente a necessidade de uma abordagem sistêmica e da percepção do mundo vivo como uma rede de relações e conexões em contínuo processo de retroalimentação (feedback) no processo de restauração ecológica de ecossistemas degradados.

Assim, pode-se caracterizar um sistema natural como organizacionalmente fechado, pois os componentes do sistema estão interagindo entre si, porém, é aberto ao fluxo de matéria e energia, recebendo entradas (inputs) de matéria e energia, transformando-as e gerando saídas (outputs). Na modelagem de ecossistemas naturais é necessário analisar os fluxos e as transformações de determinadas entradas, tais como: água, sedimentos, propágulos, solo, matéria orgânica, nutrientes, radiação solar, entre outros (AUMOND, 2003; AUMOND, 2007; AUMOND, 2009; AUMOND et al., 2012).

Já um ecossistema degradado, que teve sua vegetação suprimida, como nos casos de mineração e pecuária, pode ser visto como um sistema organizacionalmente aberto, com uma estrutura dissipativa, em que fluem matéria e energia constantemente, mas o sistema mantém sua estrutura estável (AUMOND, 2007). Nesta percepção, um sistema degradado sofreu uma interrupção nas relações entre seus componentes, deixando de exercer suas propriedades emergentes. Brown e Lugo (1994) afirmam que as entradas (inputs) em um ecossistema degradado não são mais capazes de repor as perdas de matéria orgânica do solo, nutrientes e biomassa e estoque de propágulos. Isto se deve à baixa retenção interna de matéria e energia no ecossistema degradado (AUMOND et al., 2012).

$$
\text { Para Parrotta (1992), ecossistemas }
$$

degradados são aqueles caracterizados por solos empobrecidos e erodidos e que apresentam instabilidade hidrológica, produtividade primária e diversidade biológica reduzida.

Blum (1998), ao introduzir o conceito de energia armazenada nos sistemas, propôs que ambiente degradado seja caracterizado pela perda dessa energia. Ao se comentar a definição de Blum (1998) e Kobiyama et al. (2001), ao assumirem que as funções e usos do solo têm como base a sua energia armazenada, sugere-se que a degradação do solo é igual à perda de suas funções e usos. Assim, nas avaliações de ecossistemas degradados e da sua recuperação, deve-se considerar o nível de energia do sistema. Ainda que a maioria dos conceitos de áreas degradadas esteja relacionada ao solo, entendese aqui, que neste deve estar englobado também a água, o ar e os organismos. A degradação decorre de processos e fenômenos do meio ambiente, naturais ou antropogênicos que prejudicam as atividades de um ou mais organismos. A entropia é a sujeira no sistema, que resulta da desarmonia dos processos envolvidos. Área degradada é aquela que apresenta maior entropia do que um ambiente em equilíbrio dinâmico (PARROTTA, 1992).

\section{Aplicação de rugosidades no solo como técnica nucleadora para restauração ecológica}

A heterogeneidade ambiental já é conhecida por diversos autores como componente principal nas florestas de fundo de vale nos EUA (OOSTING, 1942; BUCHHOLZ, 1981; HARDIN e WISTENDAHL, 1983) e nas florestas savânicas e semideciduais do Brasil (OLIVEIRA-FILHO et al., 1994).

Vários autores já comprovaram que a heterogeneidade ambiental em ecossistemas influencia direta e indiretamente na comunidade florestal através de vários mecanismos, incluindo o recrutamento de plântulas ao longo de gradientes hidrológicos (KEDDY e ELLIS, 1985), variações no acúmulo de serapilheira do solo (TODD et al., 2000), distribuição de espécies ao longo de gradientes topográficos (CARVALHO et al., 2005) e diferenças no estabelecimento e mortalidade de plantas devido às características microclimáticas proporcionadas pelas microtopografias do solo (ELDRIDGE et al., 1991).

Moser et al. (2009) constataram uma correlação entre a concentração de nutrientes e o aumento da superfície rugosa em restauração de 


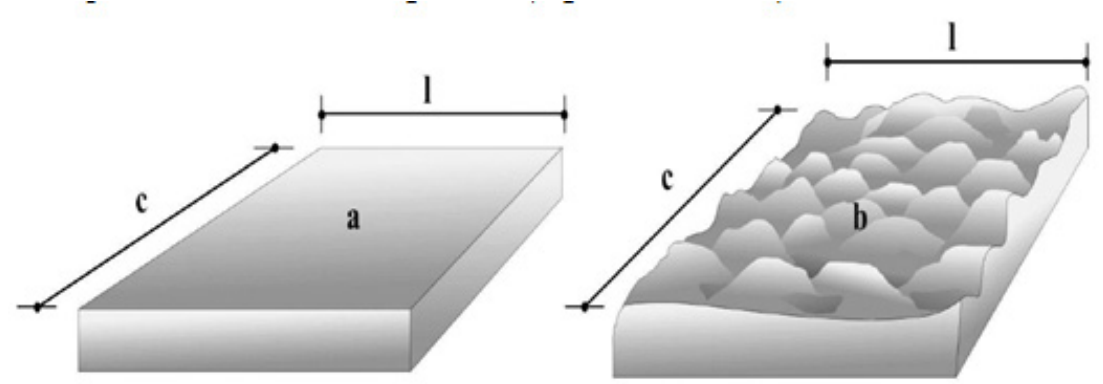

FIGURA 1: Área regular "a" cuja superfície total é igual ao produto dos lados da poligonal (comprimento (c) x largura (l)) e na área irregularizada "b" cuja superfície total é maior devido à heterogeneidade ambiental provocada pelas rugosidades.

FIGURE 1: Area regular "a" whose total area is equal to the product side of the polygonal (length (c) $\mathrm{x}$ width (1)) and the irregular area "b", whose total area is larger due to heterogeneity caused by roughening.

solos úmidos degradados. O revolvimento do solo com gradagem promove um aumento da retenção de água, da acumulação de matéria orgânica e nutrientes, estimulando a restauração desses solos.

Reis et al. (2010) sugerem que as teorias ecológicas da facilitação e nucleação constituem uma base para a restauração de ecossistemas tropicais. A restauração através das técnicas de nucleação representa uma base para o estabelecimento de comunidades que potencializam núcleos funcionais dentro da paisagem fragmentada. Assim, alguns estudos evidenciaram que as superfícies côncavas das rugosidades concentram matéria e energia e funcionam como nucleadoras, ao gerarem ilhas de diversidade, potencializando e acelerando a restauração ecológica (AUMOND, 2003; 2007; AUMOND et al., 2012; MAÇANEIRO et al., 2013).

De acordo com Aumond (2007), ao se irregularizar a superfície do solo com rugosidades, em forma de concavidades e convexidades associadas, aumenta-se a heterogeneidade física e a superfície total do ecossistema e se diminui a erosão para fora do sistema degradado (Figura 1 "a" e "b").

Desta forma, a irregularização do solo, além de acionar os fluxos de materiais, cria gradientes de temperatura, luz e umidade que não ocorrem na área regular e aumenta a superfície total do ecossistema degradado (Figura 1b). Nas áreas irregulares há uma tendência de redução do escoamento superficial devido às rugosidades que funcionam como atratores gravitacionais que retêm a água. Ao se aumentar a circulação interna de matéria (solo, água e nutrientes) pela criação de gradientes, haverá uma dissipação da energia com consequente internalização de matéria no próprio ecossistema degradado. Parte da energia da água, por exemplo, será gasta na erosão e sedimentação, dissipando a energia internamente no próprio sistema. Essa internalização no ecossistema resulta em enriquecimento ecológico e aumento da variabilidade ambiental. A retenção da água, sedimentos, nutrientes e propágulos dentro das rugosidades (superfícies concentradoras), além de conservar a matéria e dissipar energia, criam maior variabilidade ambiental que facilita $o$ estabelecimento e o aumento da biodiversidade, caso haja fonte de propágulos, induzindo a produção de biomassa e a autossustentabilidade (AUMOND et al., 2012; MAÇANEIRO et al., 2013). Esta prática reduz o escoamento superficial, aumenta a infiltração e a conservação da água e minimiza os processos erosivos, facilitando o processo de restauração ecológica (AUMOND, 2003; AUMOND, 2007; AUMOND, 2009; AUMOND et al., 2012).

Pesquisas realizadas em áreas degradadas pela mineração aplicando o método convencional de regularização da superfície, quando comparado com a aplicação das rugosidades no solo, comprovaram que as áreas regulares se comportaram como superfícies dissipadoras e as precipitações geraram mais escoamentos superficiais para fora do sistema, desencadeando mais degradação. $\mathrm{O}$ volume de água escoado para fora da área regular foi significativamente superior, correspondendo a 6,8 vezes ao da área irregular e as perdas de solo na área regular foram 4,06 vezes superiores, evidenciando uma introspecção da matéria na área irregular. O coeficiente de escoamento médio da área regular foi de 0,133 contra 0,03 da área irregular, 

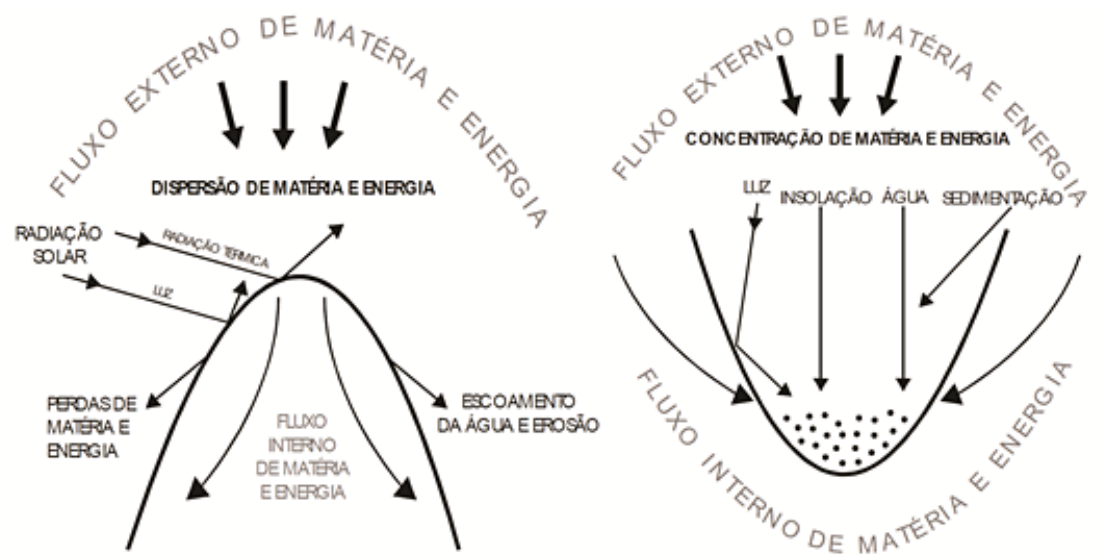

FIGURA 2: Modelos de superfícies convexa e côncava mostrando a diferença entre os fluxos externo e interno de matéria e energia divididos em superfícies dissipativas (convexa) e concentradoras (côncavas).

FIGURE 2: Models of convex and concave surfaces showing the difference between external and internal $\mathrm{f}$ lows of matter and energy dissipative surfaces divided into (convex) and concentrators (concave).

sendo 4,43 vezes maior que na área irregular. $\mathrm{O}$ fluxo de matéria e energia na área degradada, como um sistema aberto, teve um efeito negativo, tornando-se uma fonte de perdas irreversíveis e empobrecimento. Isto pode ser comprovado pela perda de solo na área irregular que correspondeu a 0,87 Mg.ha' ${ }^{-1}$.ano ${ }^{-1}$ contra um total de 3,54 Mg.ha- ${ }^{-1}$. $\mathrm{ano}^{-1}$ na área regular (AUMOND, 2007).

Na Figura 2 são apresentados os modelos de superfícies dissipadoras e concentradoras de matéria e energia do sistema em processo de restauração. Nas superfícies convexas, os fluxos externos e internos de matéria e energia levam à dissipação de energia e perdas por erosão e lixiviação. Nas superfícies côncavas, os fatores ecológicos estão voltados para dentro do sistema, levando a uma introspecção da matéria e energia tanto de origem externa como interna do sistema (AUMOND, 2007).

Ao se associar os dois modelos de preparo da superfície do solo em restauração ecológica, cria-se a complementaridade do processo (Figura 3), minimizando as perdas por erosão e lixiviação e se otimizam os ganhos de nutrientes, água, matéria orgânica, propágulos e sedimentos.

O aumento da erosão tem um correspondente direto de sedimentação e retenção nas concavidades da área. Ao se provocar a erosão e lixiviação das formas convexas, aciona-se, na mesma proporção, a sedimentação nas concavidades, dissipandose a energia do fluxo da água, criando-se, assim, a complementaridade no sistema. Com a internalização da matéria e energia no sistema, há um enriquecimento ecológico e maior variabilidade ambiental nas áreas com tratamento irregular contendo as rugosidades. O fluxo constante de matéria e energia é o mecanismo da sustentabilidade desse processo de enriquecimento e aumento da heterogeneidade ambiental.

No processo de restauração, as áreas regulares comportam-se como superfícies dissipadoras de matéria e energia e as precipitações geram mais escoamentos superficiais para fora do sistema, desencadeando mais degradação e perda de solo.

As rugosidades das áreas irregulares, além de afetarem o microclima pela retenção da água, amenização da temperatura e aumento da umidade, influenciam beneficamente também a vegetação e a fauna pela criação de habitat diferenciados e variáveis no tempo. As relações entre as rugosidades com a água e a vegetação têm a tendência de se transformarem em ilhas de diversidade que, por sua vez, desencadeiam outros circuitos de retroalimentação com os demais componentes: fauna, solo e microclima. As relações das rugosidades com a fauna mostram-se mais efetivas na restauração porque, ao reterem água e nutrientes, transformaram-se em ilhas de diversidade, potencializando o efeito de atração da fauna, pela amenização da temperatura e disponibilização de 


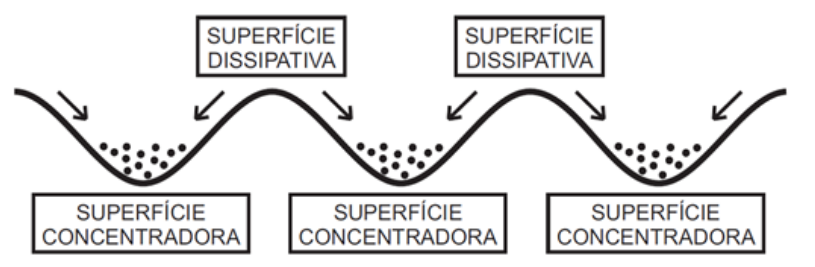

FIGURA 3: A associação de formas de superfícies côncavas (concentradoras) com formas de superfícies convexas (dissipadoras) induz ao fechamento e reorganização do sistema com introspecção dos fatores ecológicos, provocando a sua complementaridade e autonomia.

FIGURE 3: The combination of concave shapes (concentrators) with forms of convex surfaces (sinks) induces closure and reorganization of the system with insight from ecological factors, causing their complementarity and autonomy.

recursos (AUMOND et al., 2012).

Quanto maior a diversidade de microtopografias, dentro do ecossistema degradado incluindo-se formas convexas e côncavas associadas, maior será a variabilidade do padrão de luminosidade e mais intensa a circulação de nutrientes. Quanto maior for a flexibilidade dos componentes do ecossistema degradado, mais complexa será a rede e os padrões de interconexões e, consequentemente, maior será sua capacidade de busca de um novo equilíbrio dinâmico. Aciona-se, assim, o processo de retroalimentação do sistema e acelera-se a busca da autossustentabilidade.

\section{Uso de modelos sistêmicos para restauração ecológica}

Os sistemas biológicos apresentam padrões complexos de organização hierárquica e se modificam em função das interações com o meio ambiente, a vizinhança (ODUM, 1988; SOUZA e MANZATO, 2000; SOUZA e BUCKERIDGE, 2004).

Os modelos sistêmicos de análise do processo de restauração ecológica devem estar mais concentrados nas relações entre o ecossistema degradado, o ambiente e a teia complexa de interações ecológicas entre os componentes do sistema. Devem estabelecer quais as entradas ou efeitos (inputs) e quais as saídas (outputs) relevantes do ambiente geram influências consideráveis sobre o sistema. Deve haver uma concentração cuidadosa orientada para a estrutura de fluxos do sistema, estruturas de processos e mecanismos existentes entre o ecossistema degradado e o ambiente. É ainda relevante, no procedimento de análise, partir do geral para o detalhe (top-down) o que permite deslocar-se ora no âmbito do sistema mais abrangente (ambiente), ora no âmbito dos subsistemas (elementos do ecossistema degradado), sem perder de foco o conjunto das inter-relações (AUMOND, 2007; GRIFFITH, 2009; AUMOND et al., 2012).

Nos ecossistemas que tiveram o solo muito degradado, como no caso da mineração e pecuária, as variáveis ecológicas (luminosidade, temperatura, umidade do solo, escoamento da água, ciclagem de nutrientes entre outros) dificultam o aparecimento e enriquecimento da vida, e o padrão de organização será aberto, com elevada entropia (desordem), resultando em perdas progressivamente maiores e irreversíveis. A água escoa para fora do sistema, erode e carreia macro e micronutrientes, empobrecendo ainda mais o ecossistema degradado. Esta tendência para fora do sistema leva a perdas irreversíveis e a um empobrecimento progressivo do solo e homogeneização das constantes ecológicas, minimizando as possibilidades da diversidade notadamente na fase do estabelecimento vegetal (AUMOND, 2007; AUMOND, 2009; AUMOND et al., 2012).

A retenção de água será sempre menor nos ecossistemas degradados do que nos ecossistemas naturais. A insolação direta na superfície do solo provoca oscilações com extremos de temperatura. A transferência de calor solar para o meio via condução, radiação e convecção, provoca grandes oscilações térmicas no solo, seguidas de enormes perdas para o espaço (AUMOND, 2007).

Os ecossistemas preservados podem ser entendidos, aqui, como supersistemas do tipo estruturas dissipativas, em analogia à proposta de Prigogine e Stenger (1984) e Prigogine e Glansdorff (1997). Assim posto, os ecossistemas naturais apresentam uma estrutura organizacional fechada com suas populações de animais e vegetais estabelecidas. No entanto, seus componentes estão em permanente estado de mudança e o conjunto operacionaliza a cadeia alimentar cíclica com seus diferentes níveis tróficos que são alimentados 
pelo fluxo externo de matéria e energia (água, $\mathrm{CO}_{2}$ e radiação solar, entre outros). Esse modelo implica irreversibilidade do processo em que fluem continuamente matéria e energia e a estrutura do ecossistema, como um todo, permanece afastado do estado de equilíbrio. A instabilidade desse processo associada a mecanismos que permitem internalizar parte do fluxo de matéria e energia conduz à autoorganização resultante da emergência de novas estruturas que funcionam como atratores de uma complexidade crescente, advinda da não linearidade do sistema (AUMOND, 2007; AUMOND, 2009; AUMOND et al., 2012).

As plantas e outros organismos apresentam um metabolismo com dinâmica irregular devido à dinâmica não linear dos sistemas ambientais propiciando as condições favoráveis ao crescimento e adaptação ao meio (MOLLER e SWADDLE, 1998). Em ambientes com flutuações e dinâmicas caóticas, devido à instabilidade do sistema, os organismos apresentam flexibilidade adaptativa, uma vez que eles evoluíram durante milhões de anos em ambientes em constantes mudanças ambientais devido aos atratores caóticos (SHABALA et al., 1997).

A chave da compreensão do processo de restauração ecológica está na perspectiva desenvolvida por Prigogine e Stenger (1984) e Prigogine e Glandorff (1997), partindo da concepção de Bertalanffy (1975), que sugere que os seres vivos se mantêm graças ao fluxo de matéria e energia, num equilíbrio dinâmico e são mantidos pela instabilidade do sistema. Nessa perspectiva, dos sistemas denominados dissipativos, há um contínuo processo de incorporação de matéria e energia, fazendo emergir, da instabilidade uma nova estabilidade fluente, da desordem, uma nova ordem, e do desequilíbrio, um novo estado de equilíbrio instável. Destarte, ecossistemas degradados devem ser abordados como sistemas abertos com estruturas e padrão de organização em permanente estado de equilíbrio estável, sem os mecanismos básicos de resiliência. Diferentemente, os sistemas vivos funcionam afastados do estado de equilíbrio. Na ótica da teoria dos sistemas dissipativos, deve-se, então, ativar o desequilíbrio para reascender os fatores ecológicos e, consequentemente, as condições de instabilidade e o fluxo de matéria para restauração da resiliência no ecossistema degradado. Os sistemas degradados estão intimamente relacionados com o entorno, o meio ambiente.

Aumond et al. (2012) defendem o uso de modelagem para restauração ambiental em função dos padrões complexos de organização hierárquica e das interações do sistema degradado com seu meio ambiente. Os sistemas podem ser representados por modelos que são uma abstração, uma representação simplificada para facilitar a análise dos mesmos. Vale destacar a importância de que as interdependências entre o sistema e o meio ambiente fiquem claramente estabelecidas no modelo.

Para Odum (1988), a análise ecológica de um ecossistema passa necessariamente pelo uso de modelos que são versões simplificadas do mundo real. Os modelos são formulações que imitam um fenômeno real e que permitem fazer predições quantitativas, por isso devem ser estatísticos e matemáticos (formais).

A estruturação de um modelo de sistema degradado começa com a construção de um diagrama de influência (AUMOND, 2007; GRIFFITH, 2009). Para um modelo funcional de uma situação ecológica existem no mínimo quatro elementos: 1) uma fonte de energia; 2) as propriedades chamadas de variáveis de estado; 3) vias de fluxos; e 4) interações ou funções interativas. Um modelo ideal para um sistema degradado deve incluir: o espaço (os limites do sistema); os subsistemas (componentes) e um intervalo de tempo a ser considerado.

Segundo Aumond (2003), os principais componentes de um ecossistema com solo degradado que devem ser considerados num modelo teórico para restauração ecológica são: solo, água, vegetação, fauna, rugosidades e microclima. As rugosidades são variáveis do relevo que influenciam a água, a vegetação, a fauna e o solo e representam as irregularidades da superfície do solo, que alguns autores denominam como microtopografias (GUERRA, 1999; MOSER et al., 2009; ROSSELL et al., 2009; MAÇANEIRO et al., 2013). O microclima é expresso pela radiação, temperatura ambiente, temperatura do solo e umidade relativa do ar.

No diagrama de influência da Figura 4 estão representados o ambiente (vizinhança) e seus componentes e o ecossistema degradado com seus principais componentes. As linhas representam as interações e as setas representam o sentido das interações entre duas variáveis (e.g. água e solo), e podem indicar se o efeito é no mesmo sentido ou em sentido contrário. Assim, por exemplo, mais água escoando pela superfície do solo no ecossistema degradado, pode representar mais erosão no subsistema solo. 


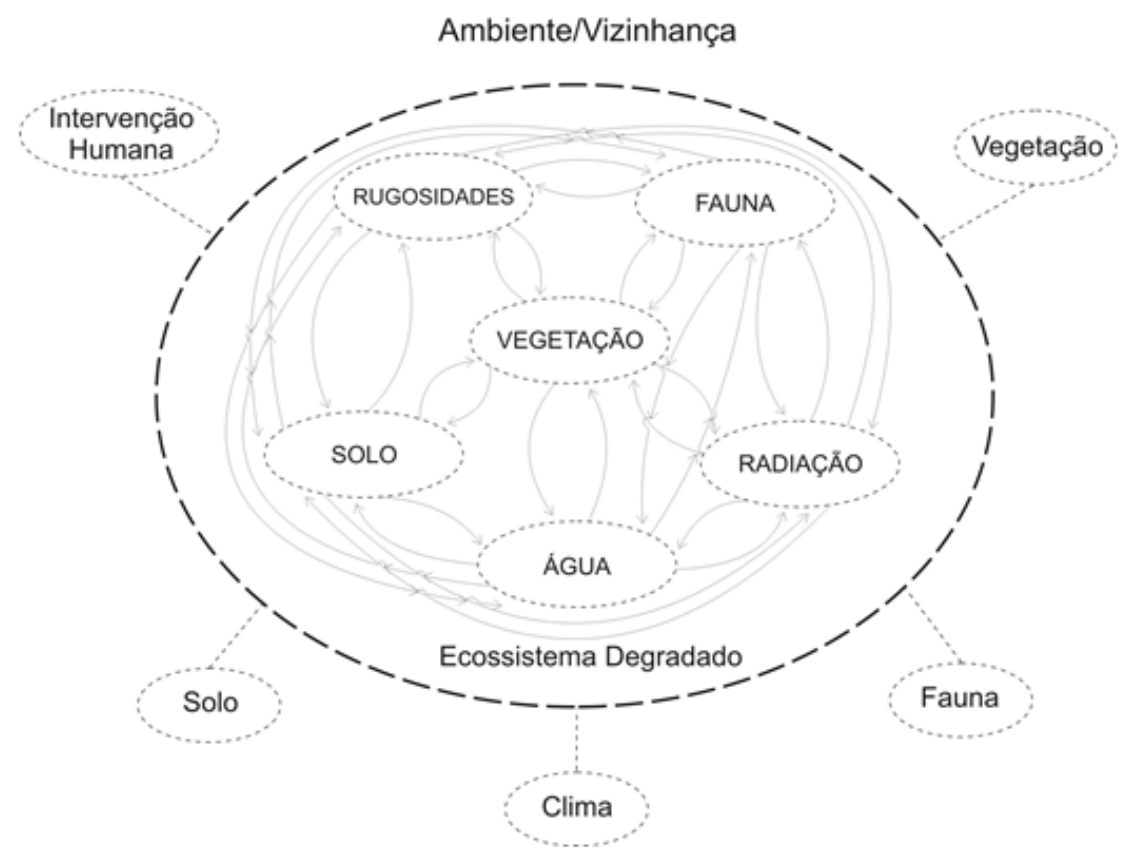

FIGURA 4: Diagrama de influência simplificado identificando os principais componentes (elementos) de um ecossistema para restauração ecológica. As linhas representam as interações entre os componentes e as setas indicam o sentido das interações entre as variáveis.

FIGURE 4: Simplified influence diagram identifying the main components (elements) of an ecosystem for ecological restoration. The lines represent the interactions between components and the arrows indicate the direction of interactions between variables.

Maior cobertura vegetal significa maior amenização ambiental microclimática devido à menor radiação solar que resulta em temperaturas do ambiente e do solo menor e maior umidade relativa do ar. As rugosidades como variações do relevo podem significar mais água, sedimentos, propágulos, solo e matéria orgânica retidos no ecossistema degradado. A água afeta o microclima pela amenização de suas variáveis, temperatura e umidade entre outros (AUMOND, 2007).

No diagrama de influência da Figura 5, os componentes do nível A, ao serem analisados no nível B, são os subsistemas do ecossistema degradado e constituem nodos inter-relacionados formando uma rede de interações complexas. Nessa representação hierarquizada, no nível $\mathrm{C}$ estão representados os componentes do subsistema solo que forma um sistema aninhado dentro do sistema maior contido no nível B.

\section{CONSIDERAÇÕES FINAIS}

Não se podem substituir os processos naturais, mas, sim, estimulá-los na busca de um novo estado de equilíbrio dinâmico. A vegetação de um determinado ecossistema em processo de restauração deve ser uma consequência de todo um conjunto de procedimentos ecológicos adequados. As espécies vegetais instalam-se mais rapidamente, intervindo com a sinergia humana em todas as etapas da compartimentação geoecológica, incluindo a irregularização morfológica do solo com criação de microtopografias (rugosidades), provocando a variação de insolação, sombreamento, umidade, erosão e sedimentação, gerando, enfim, a diversidade física e variação dos demais fatores ecológicos, como consequência da maior variabilidade de micronichos disponíveis. Assim, aumentam-se as interações e inter-relações entre os elementos que compõem o ecossistema degradado e o ambiente.

$\mathrm{O}$ desenvolvimento acelerado da vegetação em micronichos resultante da retenção de matéria e energia mantém o ecossistema em um equilíbrio dinâmico (fluente), induzindo-o a uma reorganização abiótica que oportuniza a diversidade e o estabelecimento de espécies vegetais diferenciadas. Assim, estabelece-se a biodiversidade. Da macrodesordem criam-se pequenas ilhas de 

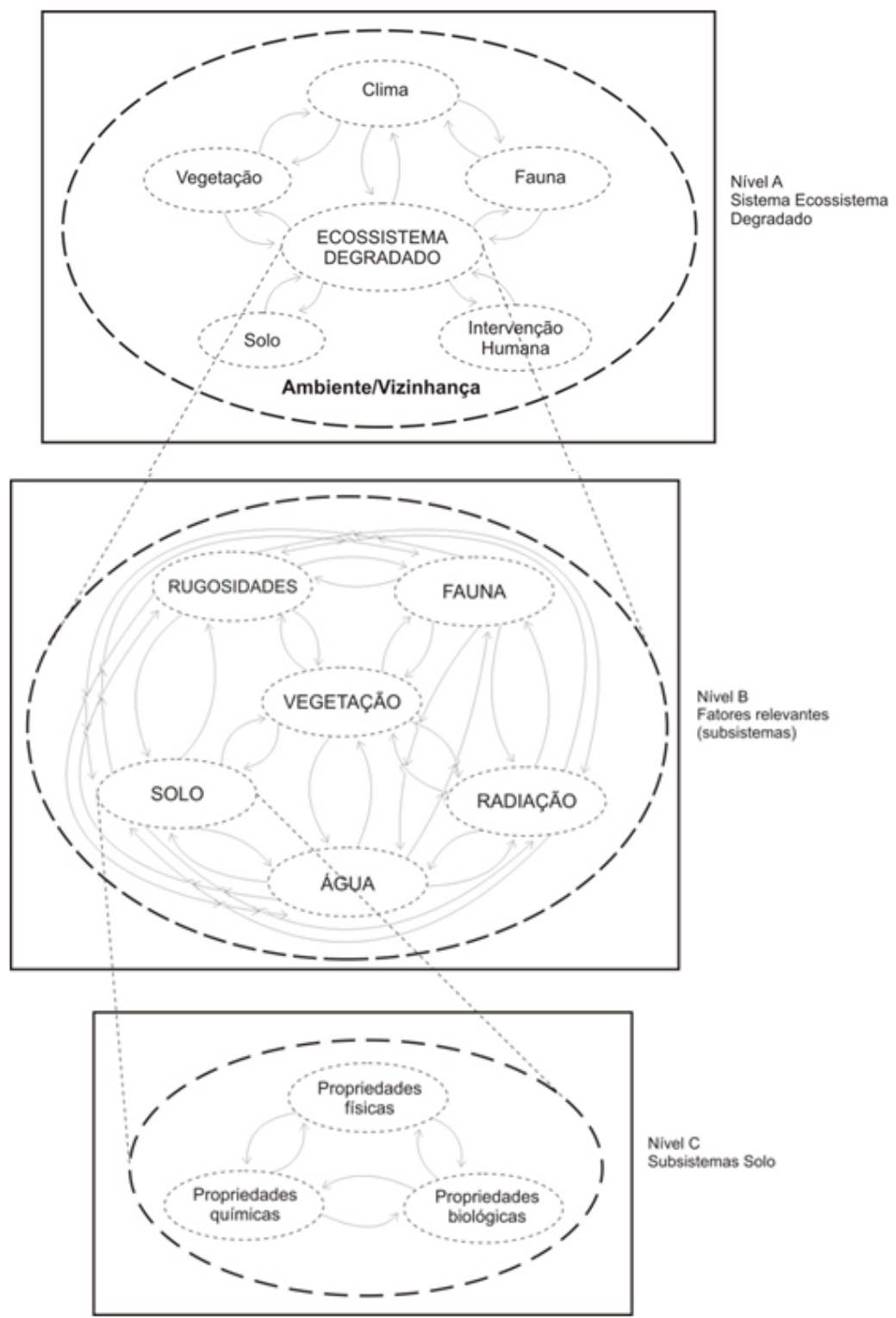

FIGURA 5: Diagrama de influência simplificado do ecossistema degradado com os componentes do ambiente (nível A). No nível $\mathrm{B}$ estão representados os elementos (subsistemas) do ecossistema degradado e no nível $\mathrm{C}$ estão representados os componentes do subsistema solo.

FIGURE 5: Simplified influence diagram of the degraded ecosystem with the components of the environment (level A). At level B, the elements (subsystems) of the degraded ecosystem are represented and at level $\mathrm{C}$ the soil subsystem components are represented.

ordem, com uma nova organização de riqueza e de diversidade que tende, devido ao fluxo de matéria e energia, a se irradiar e a se ampliar para uma organização superior num espaço geográfico maior e que acabará por envolver as superfícies côncavas e convexas. O fluxo de matéria e energia mantém o ecossistema num equilíbrio dinâmico, renovando a disponibilização de nutrientes, água e oxigênio.
Cria-se uma nova tendência à introspecção de matéria e energia que leva a uma variabilidade maior de micronichos, potencializando o ecossistema para a biodiversidade.

Precisa-se trabalhar com a perspectiva de que os ecossistemas degradados configuram-se como sistemas abertos e, na restauração ecológica, tem-se que induzir o ecossistema ao fechamento 
organizacional, mantendo-o aberto ao fluxo de matéria e energia, de forma a manter um equilíbrio dinâmico. As rugosidades do solo comportam-se como atratores gravitacionais, a semelhança do atrator estranho de Anand e Desrochers (2004), na qual o processo de restauração é difícil de se prever, mas que representa as circunstâncias necessárias para que o sistema ecológico siga sua complexa trajetória natural devido à introspecção dos fatores ecológicos e da entropia.

As superfícies côncavas das rugosidades concentram matéria e energia e funcionam como nucleadoras, ao gerarem ilhas de diversidade, potencializando e acelerando a restauração ecológica.

O modelo ecológico baseado no diagrama de influência e nos círculos de causalidade constitui-se numa ferramenta auxiliar para entender as relações de causalidade, as interações e a retroalimentação das variáveis e sua evolução espacial e temporal no processo de restauração ecológica.

\section{REFERÊNCIAS BIBLIOGRÁFICAS}

ANAND, M.; DESROCHERS, R. E. Quantification of restoration success using complex systems concepts and models. Restoration Ecology, v. 12, n. 1, p. 117-123, 2004.

ARONSON, J. et al. What Role Should Government Regulation Play in Ecological Restoration? Ongoing Debate in São Paulo State, Brasil. Restoration Ecology, v. 19, n. 6, p. 690-695, 2011.

AUMOND, J. J. Teoria dos Sistemas: uma nova abordagem para recuperação e restauração ambiental. In: SIMPÓSIO BRASILEIRO DE ENGENHARIA AMBIENTAL. Itajaí. Anais... Itajaí: Univali, p. 10-16, 2003.

AUMOND, J. J. Adoção de uma nova abordagem para recuperação de área degradada pela mineração. 2007. 265 f. Tese (Doutorado em Engenharia Civil) - Universidade Federal de Santa Catarina. Florianópolis, 2007.

AUMOND, J. J. Reflexões sobre a necessidade de uma nova abordagem na reconstrução dos ecossistemas degradados. In: TRES, D. R.; REIS, A. Perspectivas sistêmicas para a conservação e restauração ambiental: do pontual ao contexto. Itajaí: Herbário Barbosa Rodrigues, 2009. p. 45-60. AUMOND, J. J. et al. Abordagem sistêmica e o uso de modelos para recuperação de áreas degradadas. Revista Árvore, v. 36, n. 6, p. 1099-1118, 2012. BERTALANFFY, L. V. Teoria geral dos sistemas.
Brasília: Petrópolis: Vozes. 1975.

BLUM, W. E. H. Basic concepts: degradation, resilience, and rehabilitation. In: LAL, R. et al. Methods for assessment of soil degradation. New York: CRC Press, 1998. p. 1-16.

BRANCALION, P. H. S. et al. Instrumentos legais podem contribuir para a restauração de florestas tropicais biodiversas. Revista Árvore, v. 34, n. 3, p. 455-470, 2010.

BROWN, S.; LUGO, A. E. Rehabilitation of tropical lands: a key to sustaining development. Restoration Ecology, v. 2, n. 2, p. 97-111, 1994.

BUCHHOLZ, K. Effects of minor drainages on woody species distributions in a successional floodplain forest. Canadian Journal of Forest Research. v. 11, n. 3, p. 671-676. 1981.

CAPRA, F. A Teia da Vida: uma nova compreensão científica dos sistemas vivos. São Paulo: Cultrix. 1996. $256 \mathrm{p}$.

CARVALHO, D. A. et al. Distribuição de espécies arbóreo-arbustivas ao longo de um gradiente de solos e topografia em um trecho de floresta ripária do Rio São Francisco em Três Marias, MG, Brasil. Revista Brasileira de Botânica, v. 28, n. 2, p. 329-345, 2005.

DURIGAN, G. et al. Normas jurídicas para a restauração ecológica: uma barreira a mais a dificultar o êxito das iniciativas?. Revista Árvore, v. 34, n. 3, p. 471-485, 2010.

ELDRIDGE, D. J. et al. Soil-surface characteristics, microtopography and proximity to mature shrubs: effects on survival of several cohorts of atriplex vesicaria seedlings. Journal of Ecology. v. 79, n. 2, p. 357-364, 1991.

ENGEL, V. L.; PARROTTA, J. A. Definindo a restauração ecológica: tendências e perspectivas mundiais. In: KAGEYAMA, P. Y.; OLIVEIR, R. E.; MORAES, L. F. D.; ENGEL, V. L.; GANDARA, F. B.. Restauração Ecológica de Ecossistemas Naturais. Botucatu: FEPAF, 2008. p. 1-26.

GRIME, J. P. Biodiversity and Ecosystem Function: The Debate Deepens. Science, v. 277, n. 5330, p. 1260-1261, 1997.

GRIFFITH, J. J.; TOY, T. J. O modelo físico-social da recuperação ambiental. Brasil Mineral, v. 22, n. 242, p. 166-174, 2005.

GRIFFITH, J. J. Pensamento sistêmico aplicado ao ensino de recuperação ambiental e restauração ecológica. In: TRES, D. R.; REIS, A. Perspectivas sistêmicas para a conservação e restauração ambiental: do pontual ao contexto. Itajaí: Herbário Barbosa Rodrigues, 2009. p. 31-44. 
GUERRA, A. J. T. O inicio do processo erosivo. In: GUERRA, A. J. T.; SILVA, A. S.; BOTELHO, R. G. M. Erosão e conservação dos solos: conceitos, temas e aplicações. Rio de Janeiro: Bertrand Brasil, 1999. p. 17-55.

HARDIN, E. D.; WISTENDAHL. W. A. The effects of floodplain trees on herbaceous vegetation patterns, microtopography and litter. Bulletin of the Torrey Botanical Club. v. 110, n. 1, p. 23-30, 1983. HOBBS, R. J.; HARRIS, J. A. Restoration Ecology: repairing the Earth's ecosystems in the new Millennium. Restoration Ecology, v. 9, n. 2, p. 239-246, 2001.

KEDDY, P. A.; ELLIS, T. A. Seedling recruitment of 11 wetland plant species along a water level gradient: shared or distinct responses?. Canadian Journal of Botany. v. 63, n. 10, p. 1876-1879, 1985. KOBIYAMA, M. et al. Áreas degradadas e sua recuperação. Informe Agropecuário, Belo Horizonte. v. 3, n. 25, p. 10-17, 2001.

MAÇANEIRO, J. P. et al. Aplicação de uma técnica alternativa de manejo físico do solo no cultivo de Eucalyptus grandis W.Hill (Myrtaceae). Revista Árvore, v. 37, n. 1, p. 9-18, 2013.

MACIEL, J. Elementos de teoria geral dos sistemas. Petrópolis: Vozes. 1974.

MOLLER, A. P.; SWADDLE, J. P. Asymmetry, Developmental Stability, and Evolution. Oxford: Oxford University Press, 1998. 304 p.

MORAES, L. F. D. et al. Restauração florestal: do diagnóstico de degradação ao uso de indicadores ecológicos para o monitoramento das ações. Oecologia Australis, v. 14, n. 2, p. 437-451, 2010. MOSER, K. F. et al. The Influence of Microtopography on Soil Nutrients in Created Mitigtion Wetlands. Restoration Ecology, v. 17, n. 5, p. 641-651, 2009.

NAEEM, S.; LI, S. Biodiversity enhances ecosystem reliability. Nature, v. 390, n. 6659, p. 507-509, 1997.

O'CONNOR, I. M. The art of system thinking: Essencial skills for creativy and problem solving. London: Thorsens. 1997. 264 p.

ODUM, E. P. Ecologia. Rio de Janeiro: Guanabara Koogan, 1988. 434 p.

OLIVEIRA-FILHO, A. T. et al. Estrutura fitossociológica e variáveis ambientais em um trecho da mata ciliar do córrego dos Vilas Boas, Reserva Biológica do Poço Bonito, Lavras (MG). Revista Brasileira de Botânica, v. 17, n. 1, p. 6785, 1994.
OOSTING, H. J. An ecological analysis of the plant communities of Piedmont, North Carolina. American Midland Naturalist. v. 28, n. 1, p.1-126, 1942.

PARROTTA, J. A. The role of plantation forests in rehabilitating degraded tropical ecosystems. Agriculture, Ecossystems and Environment, v. 41, n. 2, p. 115-133, 1992.

PRIGOGINE,I.;GLANSDORFF,P.Termodynamic Theory of Structure, Stability and Fluctuations. Wicey: Nova York. 1997.

PRIGOGINE, I.; STENGER, I. Order out of Chaos. Bantam: Nova York. 1984.

REGENSBURGER, B. et al. Integração de técnicas de solo, plantas e animais para recuperar áreas degradadas. Ciência Rural, v. 38, n. 6, p. 1773-1776, 2008.

REIS, A. et al. Nucleation in tropical ecological restoration. Scientia Agricola. v. 67, n. 2, p. 244-250, 2010.

ROSSELL, I. M. et al. Succession of a Southern Appalachian Mountain Wetland Six Years following Hydrologic and Microtopographic Restoration. Restoration Ecology, v. 17, n. 2, p. 205-214, 2009. SCHLINDWEIN, S. L. Do sistema de restauração ambiental à restauração ambiental sistêmica: orquestrando uma conversa sistêmica sobre restauração ambiental. In: TRES, D. R.; REIS, A. Perspectivas sistêmicas para a conservação e restauração ambiental: do pontual ao contexto. Itajaí: Herbário Barbosa Rodrigues, 2009. p. 17-29. SER. Society for Ecological Restoration International. Princípios da SER International sobre a restauração ecológica. Versão 2. 2004.

SHABALA, S. et al. Observations of bifurcation and chaos in plant phisiological responses to light. Australian Journal of Plant Phisiology, v. 24, n. 1, p. 91-96, 1997.

SOUZA, G. M.; BUCKERIDGE, M. S. Sistemas complexos: novas formas de ver a Botânica. Revista Brasileira de Botânica, v. 27, n. 3, p. 407419, 2004.

SOUZA, G. M.; MANZATTO, A. G. Hierarquia auto-organizada em sistemas biológicos. In: D’Ottaviano, I. M. L.; Gonzáles, M. E. Q. Autoorganização: estudos interdisciplinares. CLE/ UNICAMP, Campinas, v. 30 p. 153-173, 2000.

TODD, M. C. L. et al. Litter cover as an index of nitrogen availability in rehabilitated mine sites. Australian Journal of Soil Research. v. 38, n. 2, p. 423-434, 2000. 